\title{
Component approach to the translation of geometric models
}

\author{
A.D.Filinskikh \\ alexfil@yandex.ru \\ Nizhny Novgorod State Technical University n.a. R.E. Alekseev, Nizhny Novgorod, Russia
}

\begin{abstract}
The article considers the issue of different approaches to assessing the transfer of geometric models of assembly units between different professional software environments of different vendors. A model for calculating the metric of the volume of data loss and the calculation of the relative volume of manual recovery of geometric models after their translation are considered. For evaluation, a hierarchical structure of the parameters of geometric models is used, as well as a graph of parameters, based on which structural weighting coefficients are calculated. Algorithms of the considered approaches are described, their differences are considered. The assembly unit of a machine-building unit was constructed in the Autodesk Inventor Professional software product. Based on the parameters of the obtained geometric model, the corresponding parameter graphs were constructed and calculations were made for each proposed approach to data translation. The model was exported and the resulting files were analyzed. Weights were calculated that reflect expert preferences and structural features and are determined in accordance with the theory of rational choice. The assembly model was translated into the KOMPAS-3D geometric modeling environment. Based on the data obtained in different formats, an analysis was made and parameter distortion coefficients were obtained and the relative amount of parameter data losses was estimated when transferring the geometric model from the professional software environment Autodesk Inventor Professional to the KOMPAS-3D environment. Conclusions are made and recommendations are given on the possibility of applying the component approach to the translation of geometric models in professional software environments.
\end{abstract}

Key words: geometric model, data translation, component approach, metric, parameter graph, structural weight.

\section{Introduction}

To date, domestic industrial enterprises are in a situation of some uncertainty associated with the political and economic situation in the country. On the one hand, there are increasing requirements for the digitalization of production processes, their improvement and transition to the Fourth Industrial Revolution e, on the other hand, the requirements for software that accompanies ubiquitous production processes, and, in particular, the import substitution program. At different levels, these problems are viewed differently. But in any case, regardless of the decisions made, a huge financial injections are required. The transition from one software product to another, regardless of the reasons, for each enterprise can be very painful. This includes software costs, staff training, updating the computer equipment fleet, and, of course, the possible loss of previously accumulated material. The ability to use previous developments to modernize or create new products increases the speed of work and reduces the time for issuing finished documentation [1].

Professional software environments (PPP) for creating and working with geometric models (GM) have been and remain the subject of competition from different manufacturers who offer their technologies in the global market for the development of products, objects of architecture and construction, infrastructure, etc. Undoubtedly, this competition leads to a continuous increase in the accuracy of design objects, the convenience of working with them and other factors [2,3] that positively affect the productivity of production processes.

Unfortunately, once choosing a system of geometric modeling, it is quite difficult for production enterprises to switch to other products, and an incorrect assessment of future costs can lead to serious consequences [4].

\section{Evaluation methods}

\section{Assemble model}

In one of the methods for evaluating data transfer based on the structure of their parameters [5], the process of data transfer in the form of assembly units from one software product to another is considered. In this methodology, the assembly unit is considered as a whole, not taking into account the individual parts or other components that are present in the assembly unit. When calculating the transmission coefficient, only functionally oriented and size-oriented parameter metrics [6] of the general assembly are taken into account.

$$
V_{Z, S_{1} \rightarrow S_{2}}=\sum_{i=1}^{n} L_{i} k_{i, Z, S_{1} \rightarrow S_{2}}
$$

where $V_{Z, S_{1} \rightarrow S_{2}}$ - metric of the amount of data loss during transmission and recovery of GM in professional software environments from $S_{1}$ to $S_{2}$ in $Z$ format; $L_{1}$ dimensionally-oriented estimation of the transfer and restoration of a GM parameter; $\mathrm{k}_{\mathrm{i}, \mathrm{Z}, \mathrm{S}_{1} \rightarrow \mathrm{S}_{2}}$ - relative amount of loss of parameter data during transmission of GM in professional software environments from $S_{1}$ to $S_{2}$ in $Z$ format.

The set of GM parameters with this approach is wider than the set of parameters when transmitting single models, because When evaluating the transfer and recovery, the parameters of the assembly unit are taken into account. A functionally-oriented assessment for calculating the information metric for the transfer and restoration of GM in faculty depends not only on the set of parameters, but also on the weighting coefficients of these parameters in the graph of the hierarchical structure of the parameters (Fig. 1).

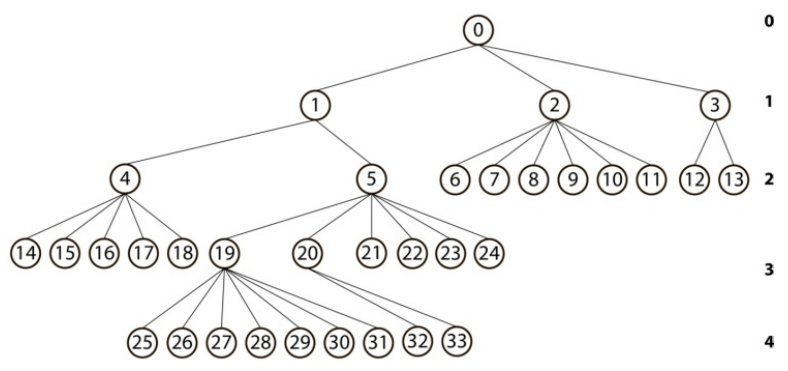

Fig. 1. Hierarchical structure of GM parameters 
The assembly parameters are at the upper levels (tiers) of this graph (element 4 in Fig. 1), as a result of which their structural weighting coefficients of the parameters of the complexity of recovering the GM parameters will have significant weight [7]. Taking into account the opinions of experts on the importance and complexity of restoring assembly parameters, we get a fairly serious effect on the parameter $\mathrm{k}_{\mathrm{i}, \mathrm{Z}, \mathrm{S}_{1} \rightarrow \mathrm{S}_{2}}$ through the manual recovery volume adjustment factor and structural weighting factors.

$$
k_{i, Z, S_{1} \rightarrow S_{2}}=w_{i}^{\ni \mathrm{c}} p_{i, Z, S_{1} \rightarrow S_{2}} \text {, }
$$

where $k_{i, Z, S_{1} \rightarrow S_{2}}$ - relative loss of parameter data during transmission of GM in professional software environments from $S_{1}$ to $S_{2}$ in $Z$ format; $p_{i, Z, S_{1} \rightarrow S_{2}}$ - relative volume of manual parameter recovery $[0,0,5,1] ; w_{i}^{\text {эc }}-$ manual adjustment volume of manual parameter recovery.

\section{Component approach}

Consider a methodology for evaluating data transfer based on the structure of their parameters, which provides for the transfer of an assembly unit of a product in the form of a set of its components and parts. With this approach, there will be no branch with assembly parameters in the graph of the hierarchical structure of parameters, and local weights will be distributed in a completely different way, increasing the weight of the remaining parameters. Soweget:

$$
\begin{aligned}
n_{\text {сб.ем }} & >n_{\text {сб.кп }} \\
w_{i, \text { сб.ем }} & <w_{i, \text { сб.кп }}, \\
L_{\text {сб.ем }} & >L_{\text {сб.кп }}
\end{aligned}
$$

Based on the data obtained, we assume that with this approach, the relative amount of parameter data loss during GM transmission should be less, and the GM transmission coefficient should increase.

To test this hypothesis, we will carry out experimental calculations on the example of an assembly unit of a machine-building product.

\section{Algorithm}

The sequence of operations during the transfer of a assembly model.

1. Creation of GM parts included in the assembly, determination of all parameters in a software environment $S_{1}$.

2. Formation of an assembly unit with the definition of all parameters.

3. Determining the hierarchical structure of parameters, creating a graph, calculating weight coefficients.

4. Formation of a single transmitting assembly file (file export).

5. Import a GM assembly into a software environment S2.

6. Assessment of accuracy of transmission of GM parameters.

7. Restore missing or distorted parameters using S2 tools.

The sequence of operations during the transfer of the model based on the component approach:

1. Creation of GM parts included in the assembly, determination of all parameters in a software environment $\mathrm{S}_{1}$.

2. Determining the hierarchical structure of parameters, creating a graph, calculating weight coefficients.

3. Formation of a single transmitting assembly file (file export).

4. Import a GM assembly into a software environment S2.

5. Assessment of accuracy of transmission of GM parameters.

6. Restore missing or distorted parameters using S2 tools.

7. The formation of the assembly unit and all the necessary parameters through the tools of the software environment S2.

\section{Modeling}

For the experiment, Autodesk Inventor Professional, the environment of geometric parametric modeling, was chosen as the first S1 system, as one of the most popular Russian systems among domestic enterprises KOMPAS$3 D v 18$ as the $\mathrm{S} 2$ system. The geometric model is built on the basis of the assembly drawing presented in Fig. 2.

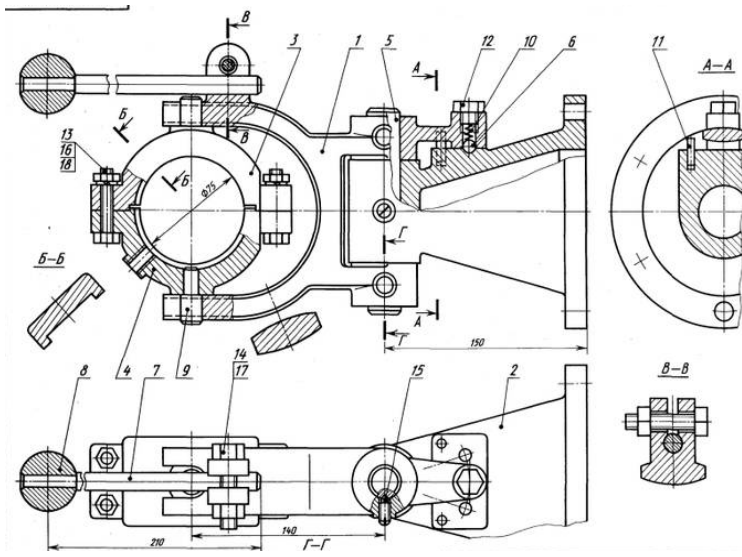

Fig 2. Drawing of section

In the Autodesk Inventor Professional environment, individual assembly parts were modeled (Fig. 3) and the assembly itself was created from the designed parts (Fig. $4,5)$.

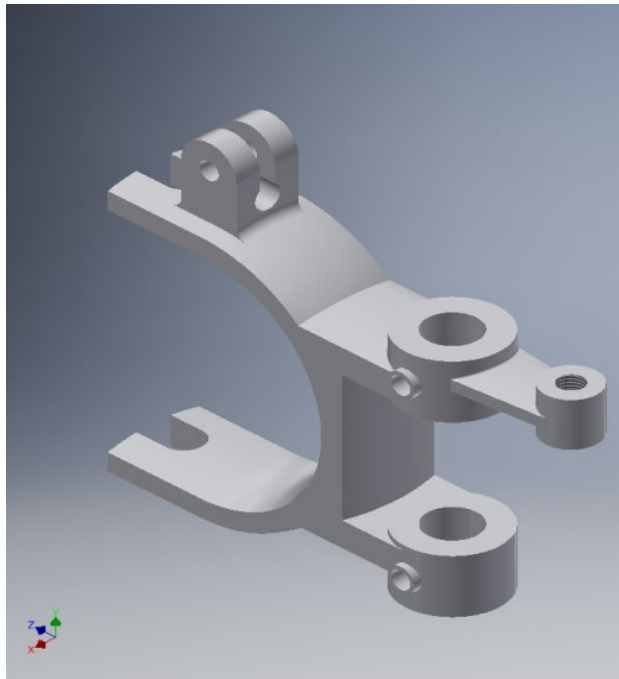

Fig. 3. An element of an assembly unit with parameters. 


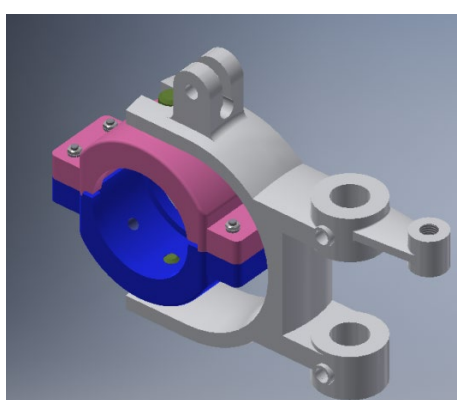

Fig. 4. 3D assembly model with parameters

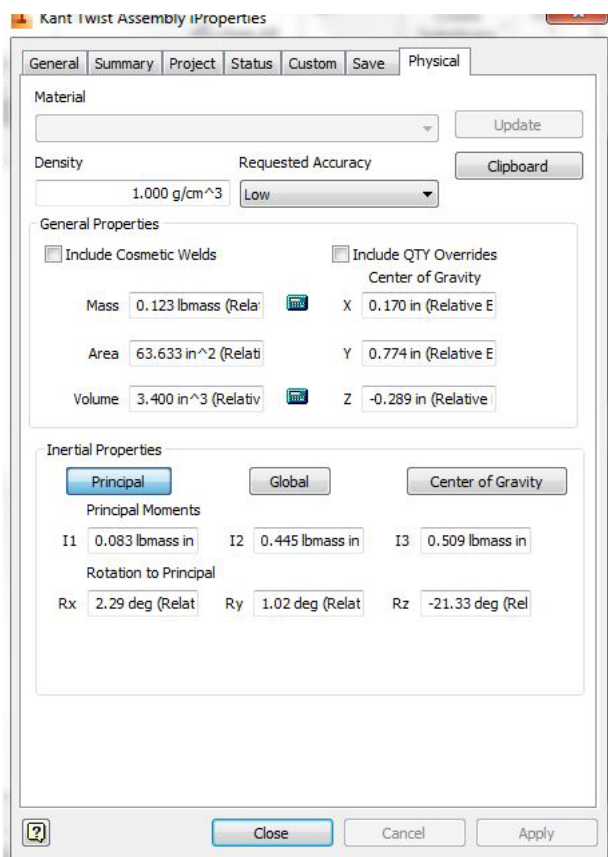

Fig. 5. Attribute information

The process of constructing a graph of parameters of a geometric model in professional software environments consists in the formation of groups of these parameters using the integration and differentiation process, based on the analysis of properties and differences inherent in the classification procedure [8]. As a result of the analysis and combining of similar properties, we can distinguish the parameters that will be included in the graph [9]. Based on the obtained graph (Fig. 1), we calculate the weight coefficients, which we will use to evaluate the transfer of GM as a single assembly unit:

1. Geometry

1.1 Assembly

1.1.1 Connection accuracy

1.1.2 Creation tree (hierarchical parameterization)

1.1.3 Assembly dependencies

1.1.4. Drawings

1.1.5 Associative

1.2 Section

1.2.1 Geometry accuracy

1.2.1.1 Node accuracy

1.2.1.2 Rib accuracy

1.2.1.3 Surface accuracy

1.2.1.4 Volume accuracy

1.2.1.5 Saving simulation type

1.2.1.6 Chamfers

\subsubsection{Connections}

1.2.2 Parameterization

1.2.2.1 Geometric parameterization

1.2.2.2 Hierarchical parameterization

1.2.3 Sketches

1.2.4. Drawings

1.2.5 Reflections

1.2.6 Thread

2. Attribute information

2.1 Material

2.2 Mass

2.3 Density

2.4 Area

2.5 Volume

2.6 Center of mass

3. File options

3.1 The possibility of using Cyrillic in the name

3.2 File size

To obtain data on the relative volume of manual parameter recovery, it is necessary to transfer the model to environment S2. To do this, we export GM to various formats for data transfer. Based on the data obtained, it is possible to analyze the sizes of the received files (Fig. 6).

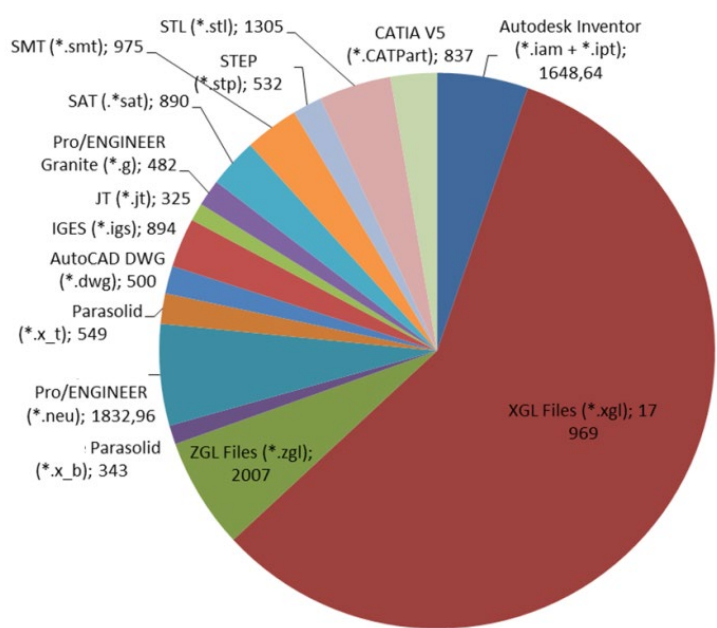

Fig. 6. Diagram of created files size

The largest file size was obtained when exporting to *.xgl $(17969 \mathrm{~Kb})$ and the smallest file size was obtained when exporting to $*$.jt $(325 \mathrm{~Kb})$, with the total size of the source files *.iam and ipt was1648,64 Kb.

Thus, the vast majority of files are not larger than the original file, with the exception of *.xgl (10.9 times more) and *.zgl (1.2 times more).

Weighting factors, which will reflect the preferences of experts and structural features, are determined in accordance with the theory of rational choice [10]. Odds are calculated using the formula:

$$
w_{i}^{\ni \mathrm{c}}=\frac{w_{i}^{\ni}}{w_{i}^{\mathrm{c}} \sum_{i=1}^{n} \frac{w_{i}^{\ni}}{w_{i}^{\mathrm{c}}}}
$$

where $w_{i}^{\ni \mathrm{c}}$ - weight coefficient reflecting expert opinions on the difficulty of reconstructing the $i^{\text {th }} \mathrm{GM}$ parameter and structural features of the graph of parameters of geometric models; $w_{i}^{\text {Э }}$ - weight coefficient reflecting only 
expert opinions; $w_{i}^{\mathrm{c}}-$ weight coefficient, reflecting only the structural features of the graph of GM parameters.

The calculation results are shown in table 1.

Table 1. The result of the calculation $\boldsymbol{w}_{i}^{\mathrm{g}}$

\begin{tabular}{|c|c|c|c|}
\hline $\boldsymbol{i}$ & $\boldsymbol{w}_{\boldsymbol{i}}^{\mathrm{c}}$ & $\boldsymbol{w}_{\boldsymbol{i}}^{\text {Э }}$ & $\boldsymbol{w}_{\boldsymbol{i}}^{\text {Эc }}$ \\
\hline $\mathbf{1}$ & 0,17 & 0,046 & 0,041 \\
\hline $\mathbf{2}$ & 0,17 & 0,036 & 0,032 \\
\hline $\mathbf{3}$ & 0,17 & 0,035 & 0,031 \\
\hline $\mathbf{4}$ & 0,17 & 0,046 & 0,041 \\
\hline $\mathbf{5}$ & 0,17 & 0,045 & 0,040 \\
\hline $\mathbf{6}$ & 0,17 & 0,046 & 0,041 \\
\hline $\mathbf{7}$ & 0,5 & 0,025 & 0,008 \\
\hline $\mathbf{8}$ & 0,5 & 0,019 & 0,006 \\
\hline $\mathbf{9}$ & 0,2 & 0,053 & 0,040 \\
\hline $\mathbf{1 0}$ & 0,2 & 0,033 & 0,025 \\
\hline $\mathbf{1 1}$ & 0,2 & 0,031 & 0,023 \\
\hline $\mathbf{1 2}$ & 0,2 & 0,043 & 0,032 \\
\hline $\mathbf{1 3}$ & 0,2 & 0,031 & 0,024 \\
\hline $\mathbf{1 4}$ & 0,08 & 0,041 & 0,078 \\
\hline $\mathbf{1 5}$ & 0,08 & 0,042 & 0,079 \\
\hline $\mathbf{1 6}$ & 0,08 & 0,040 & 0,076 \\
\hline $\mathbf{1 7}$ & 0,08 & 0,026 & 0,050 \\
\hline $\mathbf{1 8}$ & 0,14 & 0,051 & 0,055 \\
\hline
\end{tabular}

\begin{tabular}{|c|c|c|c|}
\hline $\boldsymbol{i}$ & $\boldsymbol{w}_{\boldsymbol{i}}^{\mathrm{c}}$ & $\boldsymbol{w}_{\boldsymbol{i}}^{\text {э }}$ & $\boldsymbol{w}_{\boldsymbol{i}}^{\text {Эc }}$ \\
\hline $\mathbf{1 9}$ & 0,14 & 0,052 & 0,056 \\
\hline $\mathbf{2 0}$ & 0,14 & 0,047 & 0,050 \\
\hline $\mathbf{2 1}$ & 0,14 & 0,047 & 0,051 \\
\hline $\mathbf{2 2}$ & 0,14 & 0,040 & 0,043 \\
\hline $\mathbf{2 3}$ & 0,14 & 0,025 & 0,027 \\
\hline $\mathbf{2 4}$ & 0,14 & 0,025 & 0,027 \\
\hline $\mathbf{2 5}$ & 0,5 & 0,043 & 0,013 \\
\hline $\mathbf{2 6}$ & 0,5 & 0,030 & 0,009 \\
\hline
\end{tabular}

We will determine the relative volume of manual recovery of each parameter, or the distortion coefficient of the ith parameter of the geometric model created in the professional software environment $\mathrm{S} 1$, translated into software environment $S_{2}-p_{i, S_{1} \rightarrow S_{2}}=[0,0,5,1]$. When the parameter is fully saved, the value of this coefficient is set to 0 , while the parameter is partially saved $-0,5$. If the parameter is not transmitted, the distortion coefficient takes the value 1. An example of estimating the relative volume of manual recovery of each parameter is given in table 2 .

Table 2. Estimation of the relative volume of manual recovery of each parameter in the format igs.

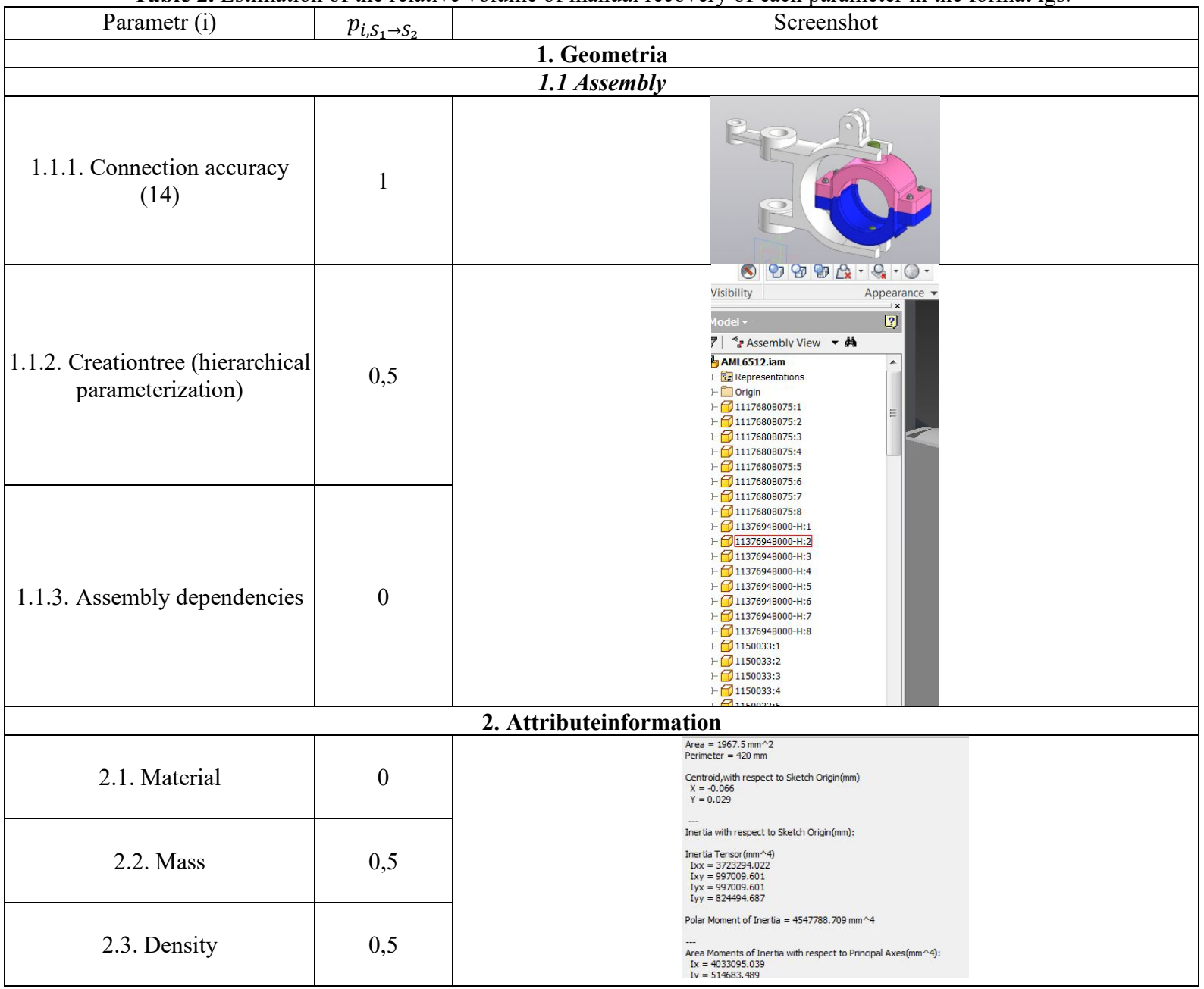

Similarly, we carry out calculations using the component approach of transferring assembly units, i.e. part transfer and assembly in S2 receiving system. We export all assembly parts in various formats and assemble the assembly unit in KOMPAS-3D v18 (Fig. 7-8).

Element 4 (assembly parameters) and its branches in the parameter graph (Fig. 1) will be absent. Accordingly, 
all weighting coefficients will be recalculated - the weighting coefficient of each parameter, reflecting the opinions of experts on the difficulty of reconstructing the $\mathrm{i}^{\text {th }} \mathrm{GM}$ parameter and the structural features of the graph of parameters of geometric models, will be greater than in the previous calculation procedure.
Thus, in the course of the work, the relative amount of loss of parameter data was obtained during the transfer of GM from the professional software environment $S 1$ to environment $\mathrm{S} 2$ in the $\mathrm{Z}$ format. A comparative table with the results is presented below (table 3 ).

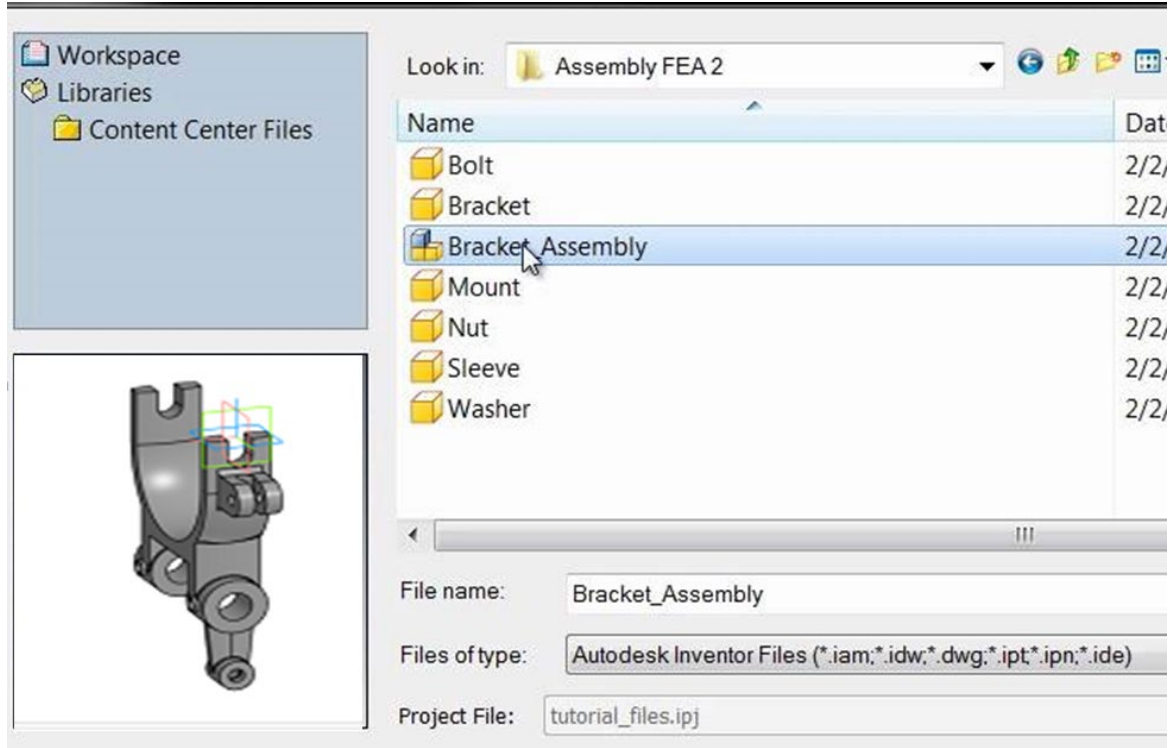

Fig. 7. The choice of parts for assembly

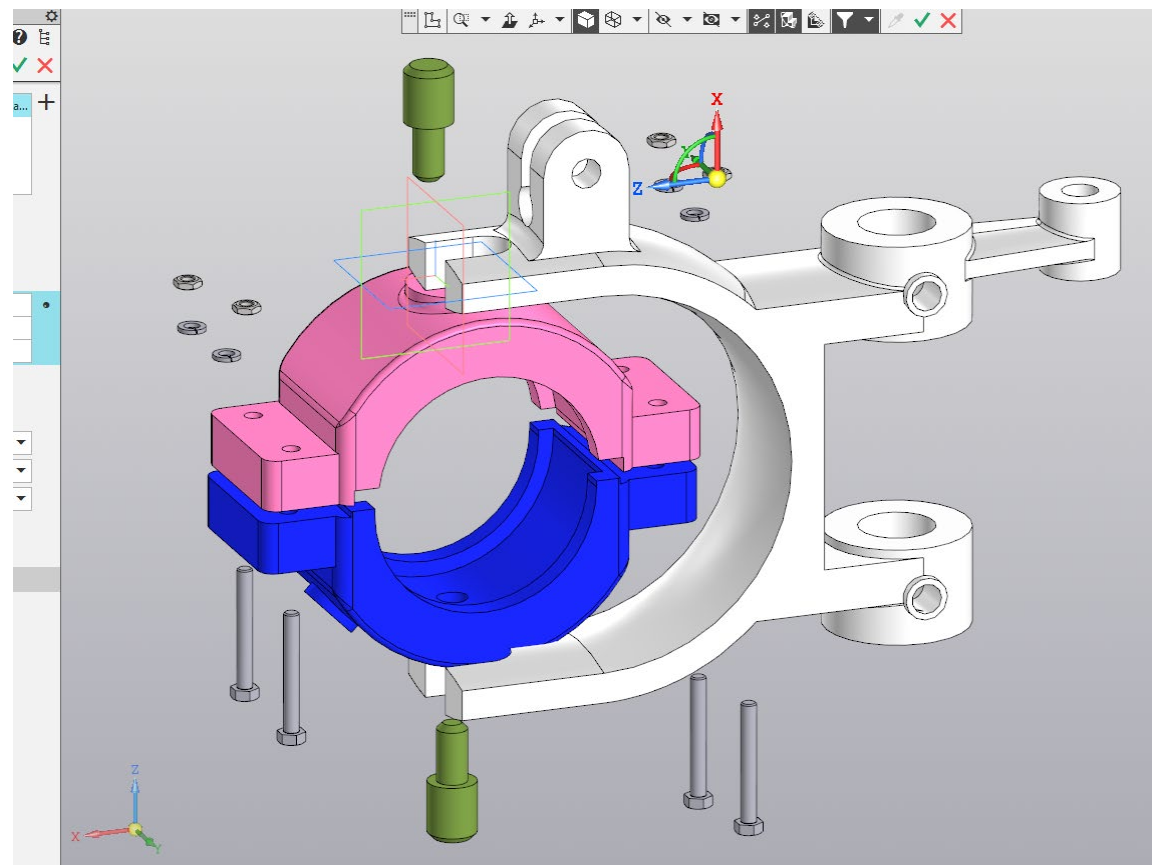

Fig. 8. The process of adding assembly components

Table 3.Datatansferevaluation \begin{tabular}{|l|l|l|l|}
\hline DataTransferRating & Value (transmission method as a assembly model) & Value (component based transmission method) \\
\hline
\end{tabular}

\begin{tabular}{|c|c|c|}
\hline$K_{d w g}$ & 0,69 & 0,77 \\
\hline$K_{i g s}$ & 0,68 & 0,76 \\
\hline$K_{j t}$ & 0,65 & 0,72 \\
\hline$K_{s a t}$ & 0,68 & 0,76 \\
\hline$K_{s t l}$ & 0,44 & 0,49 \\
\hline$K_{s t p}$ & 0,68 & 0,78 \\
\hline$K_{x \_b}$ & 0,67 & 0,73 \\
\hline$K_{x_{-} t}$ & 0,67 & 0,73 \\
\hline
\end{tabular}




\section{Conclusion:}

When transferring a GM assembly unit from one software environment to another, both methods of model transfer can be used. Eachoft he methods has its advantages and disadvantages.

1. Transmission method as a assembly model

- With this transmission method, the geometric model can be used as typical parts that do not require editing; The manual recovery time for the model is approximately $1 / 3$ of the total manual recovery time.

- If all the components of the assembly unit are created and assembled in one file (in this case, the associativity parameter is completely absent), the preservation of individual elements, their transfer and assembly in the receiver program can take significantly longer than the complete manual restoration of the assembly unit of the geometric model.

2. Component unit geometric model data transfer based on component approach.

- This method showed that the relative amount of manual recovery during the transfer of similar data will be less, respectively, the time to restore the model will be reduced.

- This method is especially relevant in the case when all components of the assembly unit are created in different files and subsequently assembled in a separate assembly file.

- The transfer of the GM in this way, despite the time required to create the assembly unit in the receiver system after the transfer of the individual components, will allow using this model not only as typical parts that do not require editing, but will also make it possible to make changes and adjustments to the assembly, i.e. . use it to build new modified products based on old models.

\section{Asknowledge}

This work was completed and published with financial support from the Russian Foundation for Basic Research, grant 19-07-00926.

\section{References}

[1] Filinskikh A.D., Byasherov A.Kh. Analysis of the transmission of parametric and graphical information based on experimental data // Herald of Belgorod State Technical University n.a. V.G. Shukhova. 2012 № 2 P. 164-166.

[2] Zakharova, A., Shklyar, A. Basic principles of data visual models construction, by the example of interactive systems for 3D visualization (2014) Scientific Visualization, 6 (2), pp. 62-73.

[3] Zakharova, A.A., Shklyar, A.V., Rizen, Y.S. Measurable features of visualization task (2016) Scientific Visualization, 8 (1), pp. 95-107.

[4] Raikin L.I. and others. About information exchange between CAD systems // Information Technology in Design and Production. 2015 № 3 (159). P. 65-72.

[5] Filinskikh A.D., Merzlyakov I.N. Assessment of geometric models based on the structure of their parameters // Information measuring and control systems. 2015 T. 13, № 3 P. 69-74.

[6] Filinskikh A.D., Raikin L.I. Functionally oriented evaluation of the transfer and restoration of geometric models // Herald of Belgorod State Technical University n.a. V.G. Shukhova. 2014 № 4 P. 176-179.

[7] Filinskikh A.D., Zarubin I.B. Methodology for assessing the completeness of regression testing with normalization by weight coefficients // Works of Nizhny Novgorod State Technical University n.a. R.E. Alekseev. 2019 № 4 (127). P. 9-16.

[8] Petrovsky A.B. Ordering and classification of objects with conflicting attributes // Artificial Intelligence News. 2003 № 4 P. 34-43.

[9] Filinskikh A.D., Sosnina O.A., Boytyakov A.A. Hierarchical space of geometric model parameters. // Herald of Belgorod State Technical University n.a. V.G. Shukhova. 2015 № 2 P. 131-135.

[10] Mikoni S.V. Theory and practice of rational choice: Monograph. M.: Route, 2004463 p.

\section{About the author}

Filinskikh Aleksandr D., Ph.D. in Technology, Associate Professor, Nizhny Novgorod State Technical University n.a. R.E. Alekseev. E-mail: alexfil@yandex.ru 